\title{
PORCENTAGEM DE PERDAS DE PESO EM CAVALOS SUBMETIDOS AO ESFORÇO DE ENDURO NA REGÃO CENTRO OESTE DO BRASIL
}

\author{
PERCENTUAL OF BODY WEIGHT LOSS OF HORSES SUBMITTED TO ENDURANCE \\ EXERCISE IN WEST CENTER OF BRAZIL
}

\author{
N. F. CONCEIÇÃ̃ ${ }^{1}$, C. J. T. FATIMA ${ }^{2}$, P. C. DUARTE ${ }^{3}$, P. S. MANHAES ${ }^{1}$ \\ A. R. TEIXEIRA NETO ${ }^{* *}$
}

\begin{abstract}
RESUMO
Objetivou-se com esse estudo avaliar o percentual de perdas de peso sofridas por cavalos submetidos a provas de enduro de 20, 40, 80 e 160 km de distância sob clima tropical na região central do Brasil (Brasília-DF). Os animais foram pesados antes, durante e após as provas com a utilização de uma balança portátil. Observou-se percentuais de


respectivamente. Tais valores foram significativamente maiores ao final da primeira etapa das provas quando comparados com o peso antes das provas, no repouso revelando importante redução de perdas na continuidade das provas, provavelmente devido ao melhor condicionamento dos animais além do livre acesso a água e feno durante os períodos obrigatórios de descanso. A utilização de balanças portáteis deve ser estimulada em provas equestres de longa duração, como uma ferramenta de monitoramento de perdas de peso imperceptíveis por veterinários durante as competições. Os resultados obtidos revelaram que mesmo animais treinados e condicionados a beber água e se alimentar durante esforços prolongados estão sujeitos a importantes perdas de peso.
\end{abstract}

PALAVRAS-CHAVE: Clima. Desidratação. Balança. Equinos.

\section{SUMMARY}

The purpose of this study was to evaluate the percentage of body weight loss suffered from horses submitted to endurance rides of 20,40, 80 and $160 \mathrm{Km}$ distance under tropical climate in West Center of Brazil (Brasilia-DF). Horses were weighted before, during and after the competition, with a portable scale. It was noticed percentual body weight losses of $3,47 \pm 1,86 \%, 5,93 \pm 4,35 \% ; 4,69 \pm 2,01 \%$ and 4,53 $22,11 \%$, in 20, 40, 80 and $160 \mathrm{Km}$ rides, respectively. These values were higher in the first weighting point, compared to basal values, with an important reduction throughout subsequent rings of rides, probably due to a better animal condition and free intake of water and forage, during the mandatory rest periods. The use of portable scales should be used for prolonged rides as a tool for veterinarians to monitor insensible body weight losses of horses in this kind of exercise. Data also revealed that even trained horses are susceptible to important body weight losses.

KEY-WORDS: Climate. Dehydration. Endurance. Scale.

\footnotetext{
${ }^{1}$ Graduação em Medicina Veterinária, FAV-UnB.

${ }^{2}$ Residente de Clínica e Cirurgia de Grandes Animais, HVet/FAV-UnB.

${ }^{3}$ Residente em Clinica de Equinos, HVet-UFMG.

${ }^{4}$ Professor Adjunto HVet/FAV-UnB. Hospital Veterinário de Grandes Animais da Universidade de Brasília, UnB, Galpão 4, Granja do Torto, 70636-200, Brasília-DF. Email: raphaeltx@unb.br. * corresponding author.
} 


\section{INTRODUÇ̃̃O}

O enduro equestre é uma competição na qual percorrem-se vários quilômetros de distância, divididos em várias fases, na qual o vencedor é o conjunto (cavalo/cavaleiro) que completa a corrida no menor tempo e de forma bem sucedida, física e metabolicamente. (TRIGO et al. 2010). É uma modalidade de esporte equestre caracterizada por um esforço aeróbico prolongado, de intensidade variável, em que o cavalo é submetido a um trabalho permanente, que exige muito dos sistemas orgânicos para a manutenção da homeostase (CARLSON, 1983).

De todas as competições eqüestres, o enduro requer a maior demanda metabólica do cavalo, ocorrendo significativa produção de energia por muitas horas (TREIBER et al., 2006). No cavalo, a eficiência metabólica dos processos que convertem energia armazenada em energia mecânica (locomoção) é baixa, sendo que a maioria da energia produzida é liberada na forma de calor (HODGSON et al., 1994). A principal forma de dissipação de calor durante o enduro é a sudorese, durante o processo evaporativo de resfriamento (TEIXEIRA-NETO, 2006), o qual leva a considerável perda de fluido corporal (CARLSON et al., 1983). . Quando há falha nos mecanismos de termorregulação, mal condicionamento animal, ou condições ambientais adversas para a realização das competições, o animal fica susceptível a desenvolver a síndrome da exaustão, que como principal agravante, pode levar o animal a óbito, em casos extremos (HODGSON et al, 1994).

Segundo CARLSON (1983), durante prova de enduro de $160 \mathrm{~km}$, estima-se uma perda de até $10 \%$ do peso seu corporal, sendo aproximadamente $90 \% \mathrm{em}$ água. Em provas de 80 e $160 \mathrm{~km}$ de distância, perdas corporais de 3 ou $4 \%$ são comuns e podem persistir após o período de uma noite de recuperação (SCHOTT et al., 1997). Teixeira Neto et a. (2006), em um grupo de cavalos, revelaram perdas maiores de $5 \%$ em provas de enduro de $100 \mathrm{~km}$ de distancia, com mais de 72 horas pós provas, para recuperação das perdas corporais de peso.

Em um estudo similar, realizado por SCHOTT (2010), entre cavalos competidores de provas variando de 80 a $160 \mathrm{~km}$, contatou-se que os animais eliminados, em geral, perderam maior porcentagem de peso corporal em relação aos animais que concluíram as provas com sucesso. Sugeriu-se também que os animais que apresentaram maior porcentagem de perda não foram os que mais perderam peso, mas sim os que não conseguiram repô-las, por meio da ingestão de água e alimentos durante a prova.

Como em atletas da espécie humana, a avaliação da perda de peso corporal de cavalos durante o exercício prolongado tem sido considerada uma forma acurada de se estimar a perda de fluidos através do suor (Kingston et al., 1997). Assim sendo, o objetivo deste trabalho foi monitorar as perdas de peso sofridas por cavalos submetidos à provas de enduro de 20, 40, 80 e 160 km de distância.

\section{MATERIAL E MÉTODOS}

Em 2011, as provas de 20, 40 e $80 \mathrm{~km} \mathrm{de}$ distância foram monitoradas de acordo com o calendário oficila da Federação Hípica de Brasília. Um total de 43 animais da raça Árabe ou cruzas-árabes, machos e femeas, que completaram as provas, foram pesados. Deste total 18, 10 e 15 animais, realizaram prova de 20, 40 e $80 \mathrm{~km}$, respectivamente. Tais provas foram realizadas com um, dois ou trêas anéis, respectivamente. Em 2012, os dados de onze animais que completaram prova official de $160 \mathrm{~km}$, com seis anéis, foram incluídos no estudo. Durante os referidos anos, na região centro oeste do Brasil (Distrito Federal) o clima foi considerado moderado $\left(17\right.$ a $28^{\circ} \mathrm{C}, 70$ a $40 \%$ umidade relativa do ar), quando as provas foram realizadas.

Todos animais foram pesados antes, durante (após completar cada anel) e ao final de cada prova, sempre após a inspeção veterinária oficial. A monitoração do peso ocorreu por meio de balança portátil (Toledo $\mathrm{MGR}^{\circledR}$ Junior) e o percentual das perdas de peso foi obtido matematicamente após as determinações do peso, em $\mathrm{kg}$. Esse projeto de pesquisa teve a aprovação do Comite de Ética em Uso Animal (CEUA-UnB 53699/2011) e todos os proprietários e cavaleiros concordaram em submeter os animais a pesagem no decorrer da pesquisa.

Para análise estatística, realizou-se após ANOVA seguida de test Tukey, com nível de significância $\mathrm{p}<0,05$, para os valores percentuais durante as provas.

\section{RESULTADOS E DISCUSSÃO}

Os resultados das pesagens dos animais no decorrer das provas estão representados na Tabela 1 . Os animais que completaram as provas de $20 \mathrm{~km}$ eram mais leves $(\mathrm{p}<0,05)$ que os demais quando comparados no momento anterior as provas. Todos apresentaram perdas significativas de peso $(\mathrm{p}<0,05)$ no primeiro ponto de pesagem, quando comparados os pesos de antes das provas. Durante as provas, o percentual de perdas de peso não foram diferentes $(p>0,05)$. Resultados similares foram observados em diversas pesquisas (BARNES et al. 2010; TEIXEIRA NETO, 2006; SCHOTT II et al. 1997). Perdas hídricas também foram notadas na urina, nas fezes e perdas significantes pela evaporação de sour pela pele e trato respiratório (SCHOTT II, 2010). Perdas de fluidos levam a um aumento da osmolalidade, causando estímulo primário a sede. Frequentemente, esta taxa de ingestão de água é associada com a ingestão de comida e pode compensar perdas hidroeletrolíticas e, alguns animais podem inclusive, terminar a prova mais pesado que na largada (KINGSTON et al. 1997; SCHOTT II et al. 1997).

Perdas corporais de peso podem ser interpretados como indicador de perdas através da sudorese. Em torno de $90 \%$ de diferenças de peso observadas durante provas de enduro podem ser atribuídas a perdas hídricas (MEYER et al. 2005; CARLSON, 1983). Tais perdas são inevitáveis e 
dependents da duração e da intensidade do esforço e condiçoes ambientais (COENEN, 2005). Em esforços realizados sob condições ambientais mais amenas (baixas temperaturas e umidade relativa do ar) ou quando em baixas velocidades, foram notados decreescimos na taxa de sudação e aumentos na ingestão de água e comida, minimizando as perdas percentuais de eso corporal (SCHOTT II, 2010).

Ocorreram percentuais de perdas corporais totais de $3,47 \pm 1,86,5.93 \pm 4.35 \%, 4.69 \pm 2.01 \%$ e $4.53 \pm$ $2.11 \%$, nas provas de 20, 40, 80 e $160 \mathrm{Km}$, respectivamente. Estudos que mensuraram perdas de peso em cavalos durante provas de enduro encontraram vlaores médios variando entre 3 a $7 \%$ ao final das provas, de acordo com Schott II et al. (1997), a depleção pode persistir por 24 horas após a prova (SAMPIERI et al. 2006). Em geral, o valor de aproximadamente $5 \%$ de perdas de massa corporea é atingido ao final das provas de enduro, independente da duração e distancia das provas. Essas perdas ocorrem a despeito da oferta de água e comida nos diversos pontos de repouso aos cavalos, com o intuito de repor fluidos, eletrólitos e energia (SCHOTT II, 2010).

Schott II (2010) também sugeriu que os animais com maiores perdas de massa corporal durante as competições não eram os perdiam maior quantidade de líquidos corporais mas aqueles que não estavam condicionados a repô-las. O presente estudo corrobora essa hipotese porque os animais que completaram as provas de 80 e $160 \mathrm{~km}$ revelaram perdas menores que os animais das provas de $40 \mathrm{~km}$ de distância, sugerindo que os animais de distâncias mais longas tiveram mais tempo para repor as perdas e sugere-se estarem mais condicionados a reposição das perdas hidroeletrolíticas. Sugere-se tambem que os animais que percorreram menores distancias, podem ter sido mais exigidos pelos cavaleiros, com velocidades medias maiores, aumentando a intensidade do esforço, com menor condicionamento para reposição dos fluidos. Barnes et al. (2010), afirmaram que os animais que não comeram nem beberam água durante as competições, estavam sujeitos a desenvolverem, por exemplo, íleo paralítico dentre outros problemas metabólicos que os levavam a eliminação das provas. Subentende-se que diminuição de apetite e da sede são sinais preocupantes que podem prevenir os animais da exaustão. Conseuqentemente, os cavaleiros e equipes de apoio devem dividir essas informações com os veterinários para controle destes eventos e tambem precisam dividir a responsabilidade de identificar os cavalos em risco de exaustão (SCHOTT II, 2010).

Tabela 1 - Peso corporal (PC) e perdas percentuais de peso (\%PPC) de cavalos de enduro, no decorrer de provas de 20 , 40, 80 e 160 km de distância no Distrito Federal, Brasil, 2011/2012.

\begin{tabular}{|c|c|c|c|c|c|c|c|c|c|c|c|}
\hline \multirow{2}{*}{$\begin{array}{c}\text { Distância } \\
\text { (km) }\end{array}$} & \multicolumn{2}{|c|}{ Repouso } & \multicolumn{2}{|c|}{ Após 20km } & \multicolumn{2}{|c|}{ Após 40km } & \multicolumn{2}{|c|}{ Após 70km } & \multicolumn{2}{|c|}{ Após 160km } & \multirow[b]{2}{*}{$\% \mathbf{P}_{\mathrm{T}}$} \\
\hline & PC & $\%$ РPC & PC & $\%$ PPC & $\mathbf{P C}$ & $\%$ PPC & PC & $\%$ PPC & PC & $\%$ PPC & \\
\hline 20 & 350.86 & 0 & 338.78 & 3.47 & & & & & & & 3.47 \\
\hline$(n=18)$ & $\stackrel{ \pm}{ \pm 1.86^{\mathrm{aA}}}$ & & $\stackrel{ \pm}{ \pm 2.45^{\mathrm{a}}}$ & $\begin{array}{c} \pm \\
1.86\end{array}$ & - & - & - & - & - & - & $\begin{array}{c} \pm \\
1.86\end{array}$ \\
\hline $\begin{array}{c}40 \\
(n=10)\end{array}$ & $\begin{array}{c}395.91 \\
\pm \\
33.22^{\mathrm{aB}}\end{array}$ & 0 & $\begin{array}{c}375.64 \\
\pm \\
25.71^{b}\end{array}$ & $\begin{array}{c}4.95 \\
\pm \\
3.92\end{array}$ & $\begin{array}{c}371.65 \\
\pm \\
25.07^{\mathbf{b}}\end{array}$ & $\begin{array}{c}5.93 \\
\pm \\
4.35\end{array}$ & - & - & - & - & $\begin{array}{c}5.93 \\
\pm \\
4.35\end{array}$ \\
\hline $\begin{array}{c}80 \\
(n=15)\end{array}$ & $\begin{array}{c}390.87 \\
\pm \\
24.27^{\mathrm{aB}}\end{array}$ & 0 & $\begin{array}{c}376.5 \\
\pm \\
24.13^{\mathbf{b}}\end{array}$ & $\begin{array}{c}3.68 \\
\pm \\
1.33\end{array}$ & $\begin{array}{c}374.23 \\
\pm \\
24.90^{\mathbf{b}}\end{array}$ & $\begin{array}{c}4.26 \\
\pm \\
2.6\end{array}$ & $\begin{array}{c}372.73 \\
\pm \\
27.22^{\mathbf{b}}\end{array}$ & $\begin{array}{c}4.69 \\
\pm \\
2.01\end{array}$ & - & - & $\begin{array}{c}4.69 \\
\pm \\
2.01\end{array}$ \\
\hline $\begin{array}{c}160 \\
(n=11)\end{array}$ & $\begin{array}{c}390 \\
\pm \\
31.40^{\mathbf{a B}}\end{array}$ & 0 & - & - & - & - & $\begin{array}{c}374.64 \\
\pm \\
28.71^{\mathbf{b}}\end{array}$ & $\begin{array}{c}3.90 \\
\pm \\
1.66\end{array}$ & $\begin{array}{c}371.95 \\
\pm \\
26.11^{b}\end{array}$ & $\begin{array}{c}4.53 \\
\pm \\
2.11\end{array}$ & $\begin{array}{c}4.53 \\
\pm \\
2.11\end{array}$ \\
\hline
\end{tabular}

Letras minúsculas, nas linhas, revelam diferença significativa $(\mathrm{p}<0,05)$ no teste Tukey Test in lines. Letras maiúsculas revelam diferença significativa $(\mathrm{p}<0,05)$ nas colunas. $\mathrm{n}$ : número de animais; $\% \mathrm{P}_{\mathrm{T}}$ : Percentual de perdas totais

\section{CONCLUSÃO}

As perdas de peso corporais foram significativamente maiores no início das provas de enduro. Embora perdas não perceptíveis ocorram, mesmo cavalos treinados devem ser condicionados a comer e beber no decorrer das competições, em pontos de parada ou descanso, para reposição de perdas hídricas evitando-se problemas clínicos relacionados a desidrtação devido ao prolongamento do esforço.

\section{REFERÊNCIAS}

BARNES, A.; KINGSTON, J.; BEETSON, S.; KUIPER, C. Endurance veterinarians detect physiologically compromised horses in a $160 \mathrm{~km}$ ride. Equine Veterinary Journal, v.42, Suppl.38, p.6-11, 2010.

CARLSON, G. P. Thermoregulation and fluid balance in the exercising horse. In: SNOW, D. H.; PERSON, S. G. B., ROSE, R. J. (Ed.). Equine exercise physiology. Cambridge: Granta Editions, 1983, p.275-309.

COENEN, M. Exercise and stress: impact on adaptive processes involving water and electrolytes. Livestock Production Science, v.92, p.131-145, 2005. 
HODGSON, D. R.; DAVIS, R. E.; MCCONAGHY, F. F. Thermoregulation in the horse in response to exercise. British Veterinary Journal, v.150, p.219235, 1994.

KINGSTON, J. K.; GEOR, R. J.; MCCUTCHEON, L. J. Rate and composition of sweat fluid losses are unaltered by hypohydration during prolonged exercise in horses. Journal of Applied Physiology, v.83, p.1133-1143, 1997.

MEYER, H.; HEILEMAN, M.; HIPP-QUARTON, A.; PEREZ-NORIEGA, H. Amount and composition of sweat in ponies. In: COENEN, M. Exercise and stress: impact on adaptive processes involving water and electrolytes. Livestock Production Science, v.92, p.131-145, 2005.

SAMPIERI, F.; SCHOTT, H. C.; HINCHCLIFF, K. W.; GEOR, R. J.; JOSE-CUNILLERAS, E. Effects of sodium chloride and potassium chloride supplementation on endurance horses competing in 80 $\mathrm{km}$ rides. Equine Veterinary Journal, Suppl.36, p.1926, 2006.

SCHOTT II, H. C.; MCGLADE, K. S.; MOLANDER, H. A.; LEROUX, A. J.; HINES, M. T. Body weight, fluid, electrolyte and hormonal changes in horses competing in 50- and 100-mile endurance rides. American Journal of Veterinary Research, Schaumburg, v.58, p.303-309, 1997.
SCHOTT II, H. C. Challenges of Endurance Exercise: Hydration and Electrolyte Depletion. Proceedings of the 17th Kentucky Equine Research Nutrition Conference, Feeding and Veterinary Management of the Sport Horse, April, 2010.

TEIXEIRA NETO, A. R. Variáveis fisiológicas e estresse oxidativo de equinos durante campeonato de enduro. 2006. 84f. Tese de doutorado. Curso de Pósgraduação em Medicina Veterinária, Faculdade de Ciências Agrárias e Veterinárias (Unesp), Jaboticabal, São Paulo, Brasil.

TREIBER, K. H.; HESS, T. M.; KRONFELD, D. S.; BOSTON, R. C.; GEOR, R. J.; FRIERE, M.; SILVA, A. M. G. B.; HARRIS, P. A. Glucose dynamics during exercise: dietary energy sources affect minimal model parameters in trained Arabian geldings during endurance exercise. Equine Veterinary Journal, Suppl.36, p.631-636, 2006.

TRIGO, P.; CASTEJON, F.; RIBER, C.; MUÑOZ, A. Use of biochemical parameters to predict metabolic elimination in endurance rides. Equine Veterinary Journal, 42, Suppl.38, p.142-146, 2010. 